\title{
Rendezvous transgastric and percutaneous sinus tract endoscopy (STE) for debridement of necrotic collections with deep retroperitoneal extension: a case series (with video)
}

\author{
Meeting presentations: Digestive Disease Week 2018
}

(우(1) $\odot$

Authors

Guru Trikudanathan, Hiba Hashmi, Ahmed Dirweesh, Stuart Amateau, Nabeel Azeem, Shawn Mallery, Martin L. Freeman

Institution

Division of Gastroenterology, Hepatology and Nutrition, University of Minnesota, Minneapolis, Minnesota, United States

submitted 28.5.2019

accepted after revision 6.1.2019

\author{
Bibliography \\ DOI https://doi.org/10.1055/a-1134-4786 | \\ Endoscopy International Open 2020; 08: E668-E672 \\ (c) Georg Thieme Verlag KG Stuttgart · New York \\ eISSN 2196-9736
}

Corresponding author

Martin Freeman, MD, Professor of Medicine, Division of

Gastroenterology, 406 Harvard St SE, MMC36, Minneapolis, MN 55455

Fax: +1-612-625-5620

freem020@umn.edu

\section{ABSTRACT}

Background and study aims Infected necrotic collections extending deep into the retroperitoneum may not be amenable solely to endoscopic necrosectomy. Rendezvous transgastric and percutaneous sinus tract endoscopic necrosectomy was conceived to maximize debridement and obviate the need for open necrosectomy or video-assisted retroperitoneal debridement.

Patients and methods Patients who underwent simultaneous rendezvous transgastric and sinus tract endoscopic transluminal necrosectomy at the same session were identified from a prospectively maintained database. Demographic data, clinical outcomes, immediate and late complications were evaluated.

Results Among 415 patients with necrotizing pancreatitis, four patients (three males, median age 47 years) underwent this intervention after a median 29.5 days following placement of percutaneous drain. Intra-procedural bleeding following dilation of percutaneous tract required placement of esophageal stent for tamponade. No patients required rescue open necrosectomy or video assisted retroperitoneal debridement. Complete removal of percutaneous drains was accomplished in all patients after a median of 78.5 days.

Conclusions Our novel approach is safe and effective and can expand the available armamentarium for management of large necrotic collection with deep retroperitoneal extension.

\section{Introduction}

Approximately $5 \%$ to $10 \%$ of patients with acute pancreatitis develop necrotizing pancreatitis (NP) [1]. Infected necrotic collections extending deep into the retroperitoneum are typically managed by percutaneous drainage because they may be inaccessible solely by traditional endoscopic transluminal techniques [2]. While percutaneous drainage and irrigation may stabilize sepsis, it may not necessarily lead to resolution of systemic inflammatory response resulting in recurrence of pain and/or infection [3]. If warranted, necrosectomy in such anato- mical locations is traditionally performed by minimally invasive necrosectomy through video-assisted retroperitoneal debridement (VARD). However, the existing percutaneous tract may also allow retroperitoneal necrosectomy using flexible endoscopy and instrumentation established for endoscopic transluminal necrosectomy [4]. This technique is referred to as sinus tract endoscopy (STE), often performed using an adult or pediatric gastroscope. Potential advantages over VARD include avoidance of iatrogenic injury associated with rigid instruments, and ability to reach very deep into the retroperitoneum in multiple directions. However, the working channel of the pe- 
diatric gastroscope may limit the extent and efficacy of necrosectomy. A rendezvous transgastric and percutaneous STE was conceived to maximize our debridement and obviate the need for more invasive VARD and open necrosectomy in selected patients with deep retroperitoneal extension.

\section{Patients and methods}

\section{Patients}

Patients with collections resulting from necrotizing pancreatitis who underwent STE over a 7-year period from 2010 to 2017 at the University of Minnesota were identified from a prospectively maintained database. All management decisions regarding initial and subsequent interventions were made in a multidisciplinary manner involving pancreatologists/therapeutic endoscopists, interventional radiologist, intensivists and surgeons using our previously described algorithm [2]. All patients considered for this study had undergone retroperitoneal percutaneous drain placement without complete resolution of symptoms due to presence of solid necrosis and/or loculations, as well as endoscopic transluminal drainage and necrosectomy in patients with appropriate anatomy. Patients who underwent simultaneous rendezvous transgastric and sinus tract endoscopic transluminal necrosectomy at the same session were included in our study. Demographic data, indication and timing, immediate and late complications and clinical outcomes were evaluated.

\section{Interventions}

Rendezvous transgastric and percutaneous sinus tract endoscopy (STE) for debridement of necrotic collections with deep retroperitoneal extension was performed as follows, entirely by an interventional gastroenterologist and without direct assistance from interventional radiology or surgery staff. All procedures were performed under general anesthesia with endotracheal intubation in the interventional endoscopy suites. Patients with large infected necrotic collections with deep retroperitoneal extension, initially underwent endoscopic ultrasound-guided cystgastrostomy with placement of $15-\mathrm{mm}$ lumen-apposing stents for endoscopic transluminal drainage. For necrotic collections with deep retroperitoneal extension, percutaneous catheter drains (PCD) (ranging from 14-20 Fr) were placed either during or within a week after endoscopic transluminal drainage based on the clinical course. PCD drains communicating with endoscopic transluminal stents were flushed with $150 \mathrm{~mL}$ of saline every 8 hours for irrigation-based debridement. Endoscopic transluminal necrosectomy was performed for debridement of solid necrotic contents every 5 to 7 days guided by the clinical course. In cases with deep, endoscopically inaccessible cavities and refractory solid necrosis, PCD catheters were serially upsized to 24 or $28 \mathrm{Fr}$ diameter to accommodate a pediatric gastroscope for subsequent sinus tract endoscopy. After maturation of the tract, the rendezvous transgastric and STE-based debridement were performed under general anesthesia under fluoroscopy ( $>$ Fig. 1). Pre-procedural intravenous prophylactic antibiotics were administered, and carbon dioxide was used for insufflation. A pediatric upper endoscope with an outer diameter of $5.9 \mathrm{~mm}$ (GIF-Q180; Olympus Inc., Center Valley, Pennsylvania, United States) was introduced through the percutaneous tract into the necrotic cavity after removing the percutaneous drain. An adult endoscope was simultaneously advanced perorally across the cystgastrostomy tract into the necrotic cavity for endoscopic transluminal necrosectomy. Endoscopic necrosectomy was performed simultaneously in an antegrade and retrograde manner using variety of accessories such as polypectomy snare, Roth nets and stone extraction balloon. The pediatric endoscope was used to loosen and push debris towards the cystgastrostomy tract for eventual retrieval with the adult endoscope. After necrosectomy was felt to be complete, the pediatric endoscope was withdrawn from the percutaneous tract and a $24 \mathrm{Fr}$ single pigtail catheter (Thalquick drain, Cook Medical, Bloomington, Indiana, United States) was replaced over a guidewire ( $\mathbf{V i d -}$ eo 1 ). The drain output was serially monitored and flushed as before. Post-intervention follow-up included inpatient or scheduled pancreas clinic visits to assess clinical status as well as interval cross-sectional imaging with contrast enhanced computed tomography and/or magnetic resonance cholangiopancreatography as needed to evaluate residual necrosis, stent position and fistulae patency. Percutaneous drains were downsized and were capped once output fell below $30 \mathrm{~mL}$ per day. They were eventually removed when cross sectional imaging after 2 weeks confirmed no residual collection.

\section{Results}

Of 415 patients with necrotizing pancreatitis managed during the study period, 114 underwent percutaneous drainage (either primary or adjuvant) for management of necrotizing pancreatitis. Among them, 19 underwent STE and four patients (three males, median age 47 years) underwent rendezvous transgastric and percutaneous STE necrosectomy. Baseline and clinical characteristics of the four patients are outlined in $>\mathbf{T a}$ ble 1. All procedures were technically successful. This intervention was performed after a median of 29.5 days after placement of percutaneous drain. There was no procedure-related mortality. Intra-procedural bleeding was encountered in one patient following dilation of the percutaneous tract, which required placement of an $18-\mathrm{mm} \times 6-\mathrm{cm}$ fully covered self-expanding metal (FCSEM) esophageal stent for tamponade and was subsequently removed after 5 days and replaced with a percutaneous drain. Worsening organ failure or development of new organ failure did not occur. There was in fact improvement in organ failure and cessation of fever after three days (average) following intervention. Patient 4 needed three additional sessions of peroral endoscopic necrosectomy. No patients required rescue open necrosectomy or video assisted retroperitoneal debridement.

Complete removal of percutaneous drains was accomplished in all patients. No seepage of fluid or necrotic debris after removal of percutaneous drain. M|median (range) time from the initial STE to complete removal of drains was 78.5 days (range 18-214) ( Table 2). 

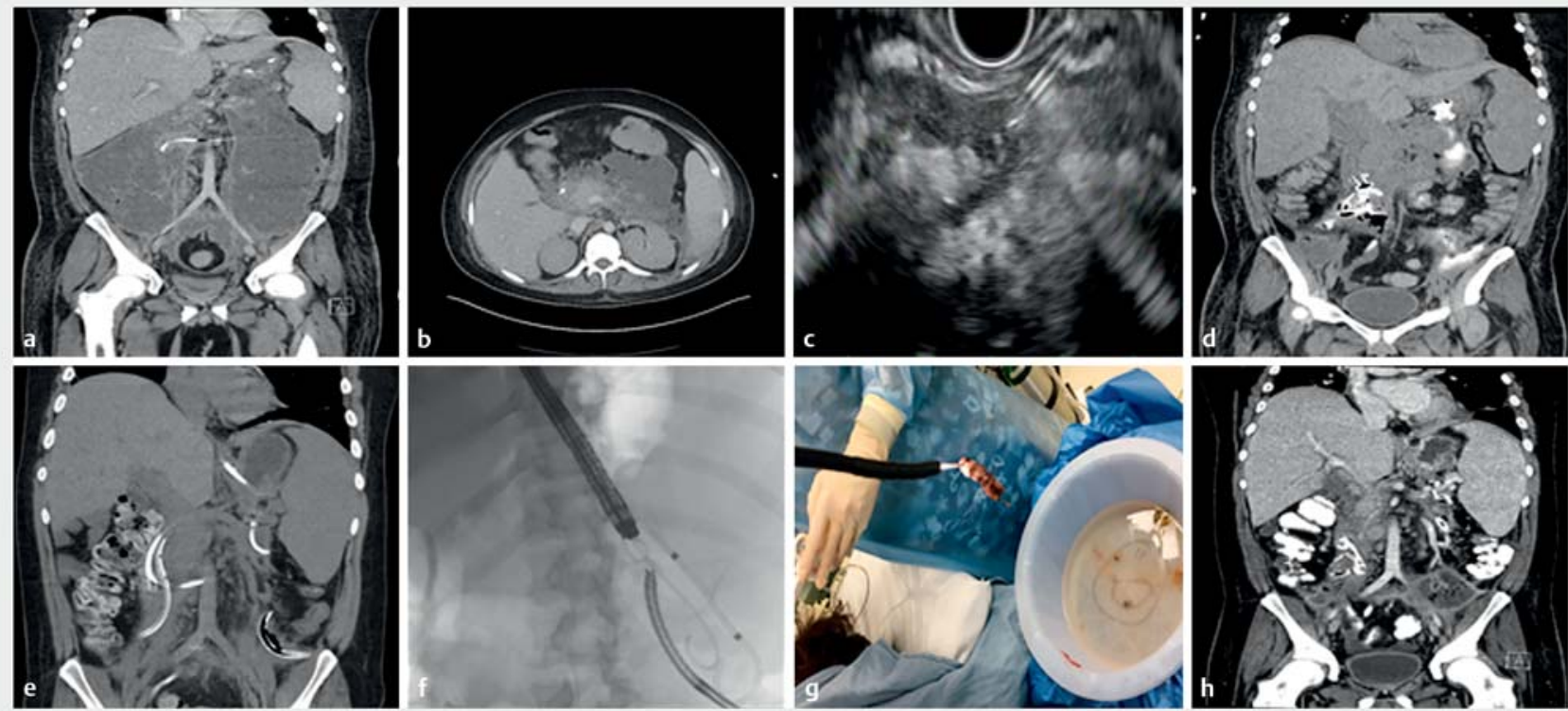

- Fig. 1 a, b CT scan showing poorly demarcated acute necrotic collection in 28-year-old male with necrotizing pancreatitis, multiorgan failure, on ventilator, dialysis for acute kidney injury with worsening tachycardia, hypotension, leukocytosis. c, $\mathbf{d}$ Endoscopic transluminal drainage performed with dual cystgastrostomies and lumen apposing metallic stents, e plus left-sided retroperitoneal percutaneous catheter drainage in tandem on day 20 after presentation, followed by two later sessions of endoscopic transluminal necrosectomy. $\mathrm{f}$ Rendezvous peroral and percutaneous sinus tract endoscopy for endoscopic necrosectomy. $\mathbf{g}$ Extensive necrotic material was debrided. $\mathbf{h}$ Resolved collections after completion of endoscopic necrosectomy.

- Table 1 Characteristics of patients managed with rendezvous transgastric/percutaneous STE.

\begin{tabular}{|c|c|c|c|c|c|}
\hline Patient & $\begin{array}{l}\text { Age } \\
\text { (years)/sex }\end{array}$ & Etiology & $\begin{array}{l}\text { Size of maximum } \\
\text { collection }(\mathrm{cm})\end{array}$ & Extent of collection & $\begin{array}{l}\text { Indication for } \\
\text { intervention }\end{array}$ \\
\hline 1 & $66 / F$ & Biliary & $9.5 \times 7$ & $\begin{array}{l}\text { Lesser sac, anterior left pararenal and perisplenic } \\
\text { spaces, inferiorly in the retroperitoneum along } \\
\text { the lateral canal fascia }\end{array}$ & $\begin{array}{l}\text { Infected necrosis with } \\
\text { sepsis }\end{array}$ \\
\hline 2 & $22 / M$ & Alcohol & $9.0 \times 5.2$ & $\begin{array}{l}\text { Bilateral deep retroperitoneal collections along } \\
\text { pararenal spaces down into pelvis and left ingui- } \\
\text { noscrotal sac }\end{array}$ & $\begin{array}{l}\text { Infected necrosis with } \\
\text { sepsis and multiorgan } \\
\text { failure }\end{array}$ \\
\hline 3 & $73 / \mathrm{M}$ & Biliary & $9.1 \times 5.8$ & $\begin{array}{l}\text { Anterior to the greater curvature of the stomach } \\
\text { and along left pararenal space }\end{array}$ & $\begin{array}{l}\text { Infected necrosis with } \\
\text { sepsis }\end{array}$ \\
\hline 4 & $28 / \mathrm{M}$ & $\begin{array}{l}\text { Alcohol and } \\
\text { hypertrigly- } \\
\text { ceridemia }\end{array}$ & $11.5 \times 5.7$ & $\begin{array}{l}\text { Bilateral deep retroperitoneal collections along } \\
\text { pararenal spaces down into pelvis, lesser sac }\end{array}$ & $\begin{array}{l}\text { Infected necrosis with } \\
\text { sepsis and multiorgan } \\
\text { failure }\end{array}$ \\
\hline
\end{tabular}

STE, sinus tract endoscopy.

\section{Discussion}

The current paradigm for intervention in necrotizing pancreatitis involves a staged multidisciplinary algorithmic approach with endoscopic transluminal drainage or percutaneous drainage as the initial step, based on the location of the necrotic collection and local expertise [5]. Recent randomized trials comparing endoscopic to surgical step-up approaches have shown that although there was no difference in mortality, endoscopic approaches resulted in shortened hospital stay and lesser indirect costs and less adverse outcomes including fistulae [6-8].
However necrotic collections that do not about the lumen of the stomach or duodenum or extend deeply into the retroperitoneum may not be amenable solely to endoscopic transluminal drainage and necrosectomy [9]. These cases warrant percutaneous drainage as a prelude to definitive necrosectomy [9]. Although percutaneous drainage is effective in temporizing sepsis in infected necrosis, solid necrotic tissue cannot be effectively evacuated via small caliber catheters. Further, when percutaneous drainage is used alone, adverse events such as pancreatico-cutaneous fistulae can occur in up to $27 \%[9,10]$. Dual modality drainage with concurrent transluminal and per- 


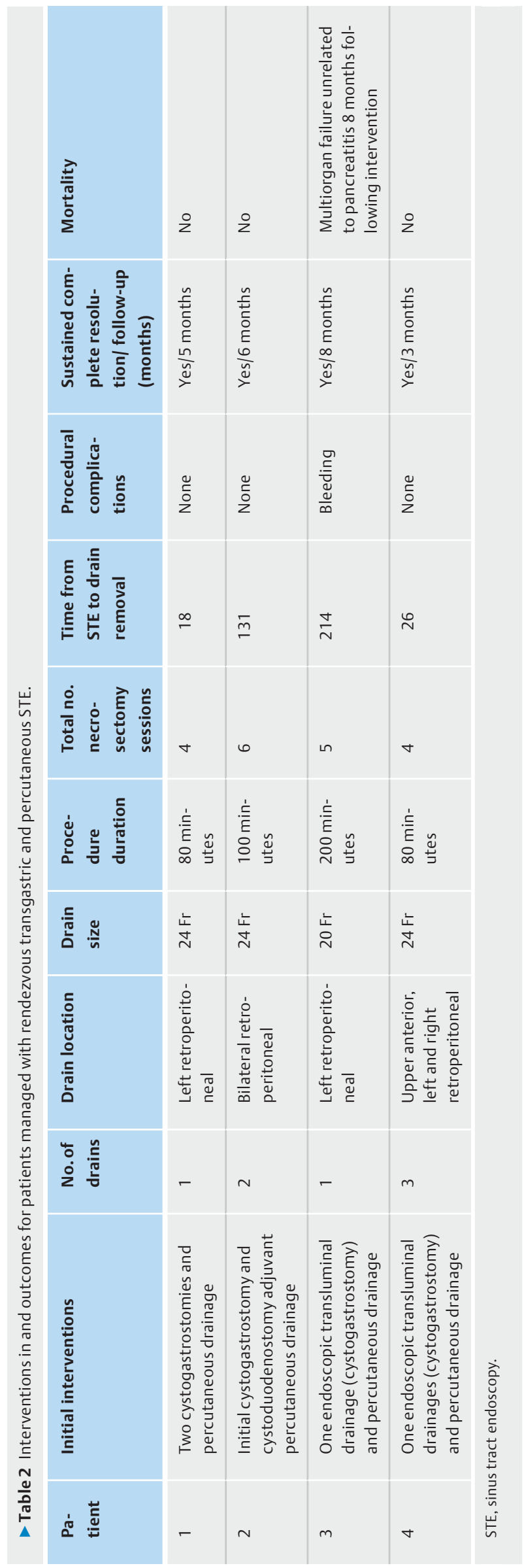

cutaneous drainage has been reported to reduce rates of pancreatic and enteric fistulae.[11] While lavage through the percutaneous catheter with egress through the transmural fistula facilitates removal of liquefied necrotic material, solid necrotic material often requires direct debridement for complete resolution.

Various forms of minimally invasive retroperitoneal necrosectomy techniques such as video-assisted retroperitoneal debridement (VARD) [12], debridement using a nephroscope [4], and mediastinoscope [13] have been described. VARD involves a 5- to 7-cm flank incision for insertion of a laparoscope, irrigation catheter, and open surgical forceps, but when compared to an endoscopic transluminal approach, recently has been reported in a randomized trial to increased rates of pancreatic fistulae and increased lengths of stay [6]. All the above instruments (laparoscope, nephroscope and mediastinoscope) are rigid, limiting maneuverability around vital structures and into deep recesses.

STE using flexible endoscope was pioneered as a technique that utilizes the existing percutaneous tract to allow retroperitoneal necrosectomy using instrumentation established for endoscopic transluminal necrosectomy $[3,4,14]$. Angulation and versatility of the flexible endoscope makes it particularly useful for accessing various extensions deep within the retroperitoneum. STE is relatively free of wound complications such as hernia because the only incision is the percutaneous drain site. Limitations include the small working channel of the pediatric endoscope which greatly restricts the extent and efficacy of debridement. The rendezvous technique of simultaneous transgastric and percutaneous sinus tract endoscopic debridement was conceived to maximize debridement volume while minimizing procedure time under sedation. It was thought to potentially obviates the need for more invasive VARD or open surgical necrosectomy.

Intra-procedural bleeding was encountered in one of our patients during percutaneous tract dilation and was treated with placement of a through-the-scope FCSEM esophageal stent for

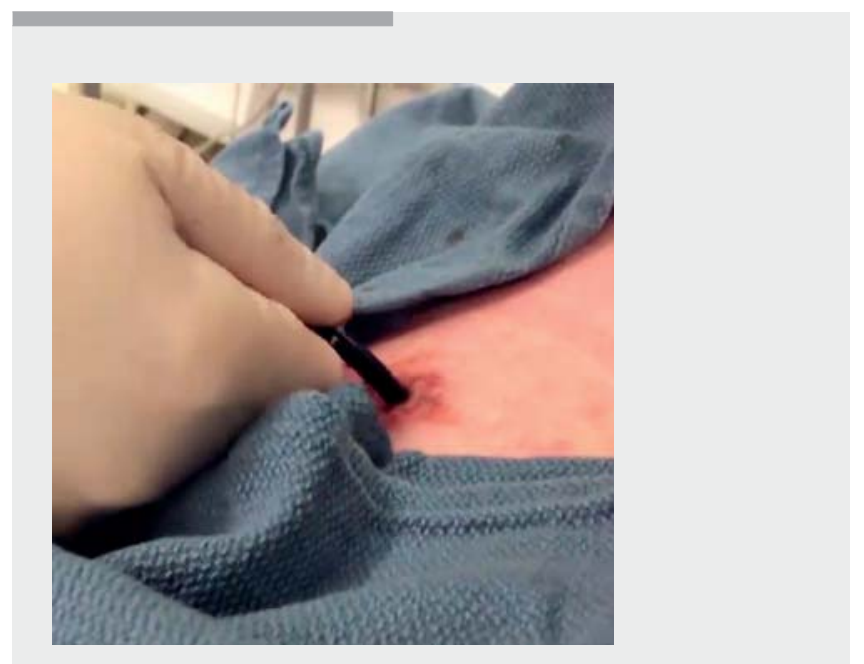

Video 1 Demonstrating the rendezvous transgastric and percutaneous sinus tract endoscopy for debridement of necrotic collections with deep retroperitoneal extension. 
tamponade. Transcutaneous endoscopic necrosectomy through FCSEM esophageal stents to secure and maintain access to the retroperitoneum has been described in small case series without a control arm and with short-term follow up [15]. While esophageal stents provide permanent access to the collection, there is a risk of pain, stent dislocation, and persistent fistula through the percutaneous sinus tract.

Simultaneous peroral and percutaneous STE for endoscopic necrosectomy essentially combines the advantages of a transgastric and a retroperitoneal approach for effective debridement. It should be considered as a sequential step-up strategy in appropriately selected patients, when percutaneous drainage fails. All forms of minimally invasive necrosectomy through a percutaneous catheter tract require a retroperitoneal route to avoid enteric leaks and dissemination of infected necrosis into the peritoneal cavity [9]. During STE, gas insufflation should be limited to minimize gas pressure within the retroperitoneum and only carbon dioxide should be used to reduce risk of air embolism [5, 14]. Advanced techniques such as rendezvous necrosectomy are best performed in close collaboration with a dedicated team of experts in pancreatic disease management including surgeons and interventional radiologists.

Limitations of the rendezvous necrosectomy techniques include small numbers of patients and lack of a comparison or control group. Larger prospective, multicenter studies would be required to validate these findings. Further studies should also compare simultaneous peroral and percutaneous approach with VARD in terms of efficacy and cost-effectiveness.

Our rendezvous transgastric and percutaneous sinus tract endoscopy approach is thus safe and effective to maximize our debridement and obviate the need for more invasive interventions in selected patients with deep retroperitoneal extension.

\section{Competing interests}

Dr. Freeman. is a consultant for Boston Scientific, Cook Medical, XLumena Corp, and Neometrics. Dr. Amateau is a consultant for Merit Endoscopy, Boston Scientific, US Endoscopy, GIE Medical, Olympus and Neurotronic and the recipient of research support from Cook Medical. Dr. Mallery is a consultant for Boston Scientific.

\section{References}

[1] Banks PA, Bollen TL, Dervenis C et al. Classification of acute pancreatitis-2012: revision of the Atlanta classification and definitions by international consensus. Gut 2013; 62: 102-111
[2] Trikudanathan G, Attam R, Arain MA et al. Endoscopic interventions for necrotizing pancreatitis. Am J Gastroenterol 2014; 109: 969-981; quiz 982

[3] Jürgensen C, Brückner S, Reichel S et al. Flexible percutaneous endoscopic retroperitoneal necrosectomy as rescue therapy for pancreatic necroses beyond the reach of endoscopic ultrasonography: A case series. Dig Endosc 2017; 29: 377-382

[4] Carter CR, McKay C], Imrie CW. Percutaneous necrosectomy and sinus tract endoscopy in the management of infected pancreatic necrosis: an initial experience. Ann Surg 2000; 232: 175-180

[5] Arvanitakis M, Dumonceau J-M, Albert ] et al. Endoscopic management of acute necrotizing pancreatitis: European Society of Gastrointestinal Endoscopy (ESGE) evidence-based multidisciplinary guidelines. Endoscopy 2018; 50: 524-546

[6] van Brunschot S, van Grinsven J, van Santvoort HC et al. Endoscopic or surgical step-up approach for infected necrotising pancreatitis: a multicentre randomised trial. Lancet 2018; 391: 51-58

[7] Bakker OJ, van Santvoort HC, van Brunschot S et al. Endoscopic transgastric vs surgical necrosectomy for infected necrotizing pancreatitis: a randomized trial. JAMA 2012; 307: 1053-1061

[8] Bang JY, Arnoletti JP, Holt BA et al. An endoscopic transluminal approach, compared to minimally invasive surgery, reduces complications and costs for patients with necrotizing pancreatitis. Gastroenterology 2018; 56: 1027-1040

[9] Trikudanathan G, Arain M, Attam R et al. Interventions for necrotizing pancreatitis: an overview of current approaches. Expert Rev Gastroenterol Hepatol 2013; 7: 463-475

[10] Freeman ML, Werner ], van Santvoort HC et al. Interventions for necrotizing pancreatitis: summary of a multidisciplinary consensus conference. Pancreas 2012; 41: 1176-1194

[11] Ross AS, Irani S, Gan SI et al. Dual-modality drainage of infected and symptomatic walled-off pancreatic necrosis: long-term clinical outcomes. Gastrointest Endosc 2014; 79: 929-935

[12] Horvath KD, Kao LS, Wherry KL et al. A technique for laparoscopicassisted percutaneous drainage of infected pancreatic necrosis and pancreatic abscess. Surg Endosc 2001; 15: 1221-1225

[13] Gambiez LP, Denimal FA, Porte HL et al. Retroperitoneal approach and endoscopic management of peripancreatic necrosis collections. Arch Surg Chic III 1998; 133: 66-72

[14] Dhingra R, Srivastava S, Behra S et al. Single or multiport percutaneous endoscopic necrosectomy performed with the patient under conscious sedation is a safe and effective treatment for infected pancreatic necrosis (with video). Gastrointest Endosc 2015; 81: 351-359

[15] Saumoy M, Kumta NA, Tyberg A et al. Transcutaneous Endoscopic necrosectomy for walled-off pancreatic necrosis in the paracolic gutter. J Clin Gastroenterol 2018; 52: 458-463 